\title{
Relationship between Body Composition and Functional Outcomes in Males with Subacute Stroke
}

\author{
Masao Hirayama, RPT ${ }^{1)}$, Yasuo Nagata, MT²), AKIMitsu Tsutou, MD ${ }^{3)}$ \\ 1)Department of Physical Therapy, Hyogo Rehabilitation Center at Nishi-Harima: 1-7-1 \\ Kouto, Shingu-Cho, Tatsuno City, Hyogo 679-5165, Japan. TEL +81 791-58-1050, \\ FAX+81 791-58-1071,E-mail:hrn_pt@hwc.or.jp \\ 2) Department of Clinical Laboratory, Hyogo Rehabilitation Center \\ 3) Department of Physical Therapy, Faculty of Health Science, Kobe University School of \\ Medicine \\ Medicine
}

\begin{abstract}
The purpose of this study was to investigate body composition focusing on motor functional capacity and to correlate body composition with functional outcomes with subacute stroke patients. The subjects of this study were 34 inpatients with hemiparetic stroke who could walk. Motor functional capacity of patients was assessed on the basis of the Brunnstrom recovery stage (BRS), and functional status was measured using the Functional Independence Measure (FIM) instrument scores and gait velocity. Body composition was assessed by bioelectrical impedance analysis. Significant correlations were observed between FIM scores and the BRS of the lower extremity or lean body mass. The correlations for body water in affected and unaffected legs with the FIM subscores were statistically significant $(p<0.05)$. The correlations for the FIM scores with BRS and body water were higher in the lower extremity than in the upper extremity. Differences in body composition were calculated between the affected and unaffected sides. There was no significant difference in body water between the affected leg and the unaffected leg, but body water in the affected arm was lower than that in the unaffected arm $(p<0.01)$. These results suggest that motor function capacity and body composition are generally related with functional status rather than the lower extremity or upper extremity.
\end{abstract}

Key words: Bioelectrical impedance analysis, Hemiplegia, Functional independence measure

(This article was submitted Oct. 3, 2006, and was accepted Mar. 27, 2007)

\section{INTRODUCTION}

Stroke is common and the major cause of physical impairment and disability in Japan today. Mortality rates from stroke have been declining, resulting in more people living with residual disability ${ }^{1)}$. The subacute recovery period may be important because changes in cardiovascular fitness might influence the restoration and maintenance of walking ability and, ultimately, have an impact on long-term disability ${ }^{2)}$. Reduced fitness may be a secondary factor that limits the transfer of walking skills obtained during rehabilitation back into the community environment ${ }^{2}$.

Several investigators have reported on stroke patients whose functional fitness was measured through tests that focused on cardiorespiratory and muscular fitness ${ }^{3,4)}$. However, research focusing on physical impairments and body composition has been limited ${ }^{5)}$.

It is not known whether body composition characteristics vary between the affected and 
unaffected legs in physically deconditioned hemiparetic stroke patients ${ }^{16)}$, and the relationship between body composition and physical performance at mild to moderate functional motor capacity, by Brunnstrom's functional motor recovery stage, of hemiparetic stroke patients. At Japanese public health centers, the level of motor function of stroke patients is evaluated using the Brunnstrom recovery stage (BRS) ${ }^{6}$, which is one of the most accepted criteria among medical doctors or physical therapists in the field of stroke rehabilitation ${ }^{7)}$.

Bioelectrical impedance analysis is an easy, low cost, and highly reliable method, making it a useful technique for studying human body composition. This method shows excellent correlation with anthropometric variables ${ }^{8)}$.

The purposes of this study were to measure body water distribution in male, subacute hemiparetic stroke patients and to investigate the correlation of body composition with functional performance. We hypothesized that body water would be lower in the hemiparetic side than in the unaffected limb in disabled hemiparetic stroke patients.

\section{MATERIALS AND METHODS}

\section{Subjects}

Thirty-four ambulatory hemiparetic stroke patients (34 males) participated in this study. All subjects were inpatients at a rehabilitation center in Japan and received physical therapy based on the motor relearning program (conventional programs). All subjects could walk in indoor at least 10 meters.

The mean age and standard deviation of the subjects were $60.4 \pm 5.4$ years old. Subjects had mild to moderate hemiparesis, including both upper and lower limbs, which were documented on physical examination. Right-side hemiparesis was observed in 22 individuals, whereas twelve had hemiparesis on the left side. The characteristics of the subjects who participated in this study are presented in Table 1.

Cerebral vascular events and affected locations were documented by a computed tomographic scan and medical diagnosis. Patients were excluded if they had any co-morbidity or disability other than stroke, such as spinal cord lesion or amputation, that would preclude gait assessment; any uncontrolled health condition for which exercise is contraindicated, such as consistent, uncontrolled
Table 1. Characteristics of the subjects $(n=34)$

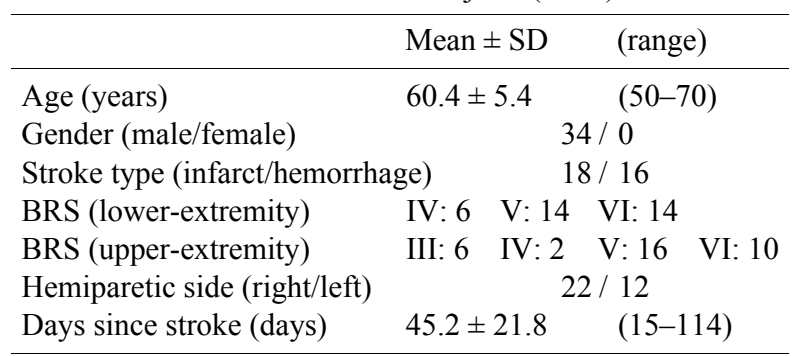

BRS: Brunnstrom recovery stage.

diabetes, or persistent, uncontrolled hypertension; significant lower limb degenerative joint disease that would interfere with gait assessment; or cognitive impairment. All patients had sufficient cognition to participate in the study and provided informed consent for this study.

\section{Measurements}

Age, height, side and location of lesion, and number of days since the stroke were recorded. Each patient was characterized using available scales for functional motor capacity, functional outcomes, and ambulation.

Motor functional capacity in patients was assessed on the basis of the BRS classification, which defines the process of functional motor recovery after stroke through 6 stages. These stages assess functional motor capacity by grading spontaneous motor recovery of both flaccid and spastic hemiparetic extremities. This test is used to evaluate physical recovery after stroke and reported in units of BRS using a numerical scoring system for items on a stage from I (flaccid paralysis) to VI (can be fully performed). And the purpose of this index is to evaluate treatment efficacy, particularly on the affected side, as well as the motor abilities of the upper and lower limbs.

Functional outcomes were evaluated by the FIM. This is a widely used clinical outcome measure and is part of the routine classification, evaluation, goal setting, and discharge criteria for stroke patients in rehabilitation settings ${ }^{9}$.

The FIM provides a useful global measure of functional independence. However, we found that patients with identical FIM scores actually differed markedly in distinct aspects of function that are targeted by particular therapies. Therefore, we used FIM subscores indicating impairment in specific domains-such as self-care, mobility, or motor 
function-as outcome measures. To obtain the FIM self-care subscore, 6 individual FIM component scores for activities (bathing, eating, grooming, dressing upper body, dressing lower body, toileting) were added. To obtain the FIM mobility subscore, 3 individual FIM component scores for transfers (bed and chair and wheelchair, toilet, tub and shower transfers) and 2 individual FIM component scores for locomotion (stairs, walking and wheelchair locomotion) were added together. The FIM motor subscore was the sum of the FIM selfcare subscore, the FIM mobility subscore, and the FIM component scores for bladder and bowel control $^{1)}$.

Gait velocity was evaluated with a stopwatch, and calculated in meters per second. The subject was asked to stand and walk with any assistive physical device during testing, if necessary, and while being guarded by and provided whatever assistance needed by a physical therapist. The walking velocity recorded was the average of two trials.

\section{Body composition}

Body composition was assessed by bioelectrical impedance in the 34 subjects, in with approximately two months of recovery from stroke.

Body weight (BW), lean body mass (LBM), body fat mass $(\mathrm{BF})$, percent body fat $(\% \mathrm{BF})$, bone mineral content (BMC), waist-hip ratio (WHR) and segmental fluid distribution were estimated by means of eight- point tactile electrode bioelectrical impedance analysis (body composition analyzer InBody 3.0, Biospace, Tokyo, Japan). Body water can be approximated to the muscle mass. The body mass index (BMI) was also calculated.

\section{Statistical analysis}

Descriptive statistics were utilized to characterize demographics. Data were analyzed on a Macintosh PowerPC using the statistical software Stat View 4.5. Data shown are mean and standard deviation.

A correlation study was conducted to establish the strength of association between measures of performance and the qualitative scales. Pearson's product-moment correlation coefficient $(r)$ was utilized to establish the correlation among the continuous data, and Spearman's rank-order was utilized for ordinal data. Student's $t$ test was used for independent observations. A value of $p<0.05$ was considered statistically significant.

\section{RESULTS}

Thirty-four males met the criteria of the study. Sixteen participants had stroke at age of $<60$ years, whereas the other eighteen had stroke at $>60$ years. The mean BRS after stroke was $5.2 \pm 0.7$, ranging from 4 to 6 , indicating that the sample consisted of individuals with mild to moderate impairment.

The mean BW, LBM, and \%BF for all subjects were $63.6 \pm 10.6 \mathrm{Kg}, 47.0 \pm 6.1 \mathrm{Kg}, 21.1 \pm 5.6 \%$, respectively. $\% \mathrm{BF}$ range from $10.8 \%$ to $36.9 \%$, indicating a wide variability in body composition in this patient population. Differences in body composition were calculated between the affected and unaffected arms and legs. There was no significant difference in body water between the affected leg and the unaffected leg, but body water in the affected arm was significantly lower than that in the unaffected arm $(p<0.01)$.

The mean gait velocity was $0.93 \pm 0.38 \mathrm{~m} / \mathrm{sec}$. Four participants had a gait velocity of $<0.45 \mathrm{~m} / \mathrm{sec}$ and four participants had a walking velocity between 0.45 and $0.75 \mathrm{~m} / \mathrm{sec}$. Seventeen participants had a velocity higher than $0.90 \mathrm{~m} / \mathrm{sec}$.

The mean FIM total score was $94.8 \pm 20.4$, indicating that the group required supervision or modified independence. There were two participants who required more than moderate assistance (FIM, <54), three who required moderate to minimal assistance (FIM, 54-72), five required minimal assistance to supervision (FIM, 72-90), and twenty-four who were classified as requiring supervision or modified independence (FIM, >90). Table 2 shows the results of body composition analysis, gait velocity and the FIM scores of the subjects.

The correlations among the outcomes variables are presented in Table 3 . The BRS of lower extremity after stroke had moderate and statistically significant associations with the FIM subscores (all $r$ values between 0.53 and $0.56, p<0.01$ ).

The Pearson's correlation for the mobility FIM score with the gait velocity was $0.38(p<0.05$, Fig. $1)$. The other FIM subscores had low correlations with the gait velocity (ranging from 0.32 to 0.36 ) that were not statistically significant.

The correlation for BMI with LBM was 0.73 $(p<0.0001)$. The Pearson's correlation for the FIM scores with LBM were between 0.37 and 0.41 $(p<0.05)$, but the correlations of the FIM scores with BMI were not statistically significant (between 
Table 2. Results of body composition, gait velocity and functional capacity $(n=34)$

\begin{tabular}{|c|c|c|c|}
\hline & & Mean \pm SD & (range) \\
\hline \multicolumn{4}{|l|}{ Body composition analysis } \\
\hline Height & $(\mathrm{cm})$ & $166.9 \pm 5.2$ & $(157.0-179.0)$ \\
\hline Body weight & $(\mathrm{kg})$ & $63.6 \pm 10.6$ & $(47.1-86.4)$ \\
\hline BMI & $\left(\mathrm{kg} / \mathrm{m}^{2}\right)$ & $22.8 \pm 3.6$ & $(17.0-30.6)$ \\
\hline LBM & $(\mathrm{kg})$ & $47.0 \pm 6.1$ & $(36.5-61.3)$ \\
\hline Body fat mass & $(\mathrm{kg})$ & $13.8 \pm 5.6$ & $(6.2-30.0)$ \\
\hline Percent body fat & $(\%)$ & $21.1 \pm 5.6$ & $(10.8-36.9)$ \\
\hline $\mathrm{BMC}$ & $(\mathrm{kg})$ & $2.75 \pm 0.28$ & $(2.26-3.39)$ \\
\hline Waist-hip ratio & & $0.90 \pm 0.05$ & $(0.81-1.06)$ \\
\hline Edema & & $0.35 \pm 0.01$ & $(0.32-0.37)$ \\
\hline Body water & (L) & & \\
\hline Affected arm & & $1.96 \pm 0.36^{*}$ & $(1.24-2.84)$ \\
\hline Unaffected arm & & $2.02 \pm 0.38$ & $(1.25-2.73)$ \\
\hline Affected leg & & $5.35 \pm 0.71$ & $(4.36-7.01)$ \\
\hline Unaffected leg & & $5.37 \pm 0.70$ & $(4.29-7.00)$ \\
\hline Trunk & & $16.4 \pm 2.1$ & $(12.0-21.1)$ \\
\hline Gait velocity & $(\mathrm{m} / \mathrm{sec})$ & $0.93 \pm 0.38$ & $(0.17-1.61)$ \\
\hline \multicolumn{4}{|l|}{ FIM } \\
\hline self-care FIM score & $(/ 42)$ & $34.1 \pm 7.6$ & $(15-42)$ \\
\hline mobility FIM score & $(/ 35)$ & $23.6 \pm 6.7$ & $(9-34)$ \\
\hline motor FIM score & $(/ 91)$ & $69.5 \pm 15.9$ & $(29-90)$ \\
\hline total FIM score & $(/ 126)$ & $94.8 \pm 20.4$ & $(36-124)$ \\
\hline
\end{tabular}

BMI: Body mass index, LBM: Lean body mass, BMC: Bone mineral content, FIM: Functional independence measure.

$*: p<0.01$ (vs. unaffected limb).

Table 3. Correlations among outcomes $(n=34)$

\begin{tabular}{|c|c|c|c|c|c|c|c|c|c|}
\hline & \multicolumn{2}{|c|}{ BRS } & \multirow[t]{2}{*}{ BMI } & \multirow[t]{2}{*}{ LBM } & \multicolumn{5}{|c|}{ Body water } \\
\hline & $(\mathrm{U} / \mathrm{E})$ & $(\mathrm{L} / \mathrm{E})$ & & & Affected arm & Unaffected arm & Affected leg & Unaffected leg & Trunk \\
\hline self care FIM & $0.36^{*}$ & $0.53 * *$ & 0.19 & $0.40^{*}$ & 0.32 & $0.36^{*}$ & $0.35 *$ & $0.39 *$ & $0.37 *$ \\
\hline mobility FIM & $0.37 *$ & $0.56^{* *}$ & 0.17 & $0.39 *$ & 0.30 & 0.31 & $0.38 *$ & $0.41 *$ & $0.34^{*}$ \\
\hline motor FIM & $0.37 *$ & $0.54 * *$ & 0.20 & $0.41 *$ & 0.33 & $0.35^{*}$ & $0.37 *$ & $0.40 *$ & $0.37^{*}$ \\
\hline total FIM & 0.29 & $0.43^{*}$ & 0.16 & $0.37 *$ & 0.29 & 0.34 & $0.36^{*}$ & $0.40^{*}$ & 0.34 \\
\hline
\end{tabular}

Spearman's coefficient was used for all variables, except between BMI, LBM, and Body water, which were based on Pearson's. BRS: Brunnstrom recovery stage, U/E: Upper extremity, L/E: Lower extremity, BMI: Body mass index, LBM: Lean body mass, FIM: Functional independence measure.

$*: p<0.05, * *: p<0.01$

\subsection{6 and 0.20$)$.}

The correlations for body water in the affected and unaffected legs with the FIM subscores ranged from 0.35 to $0.41(p<0.05)$. Body water in the affected arm had no significant correlation with any of the FIM subscores, and only the self-care and the motor FIM subscores had a significant correlation with the unaffected arm $(p<0.05)$.

The correlations of the FIM scores with BRS and body water were higher in the lower extremity than in the upper extremity.

\section{DISCUSSION}

The mean number of days elapsed since the stroke onset until admission to the rehabilitation unit supports the subacute status of the sample studied. The primary purpose of this study was to 


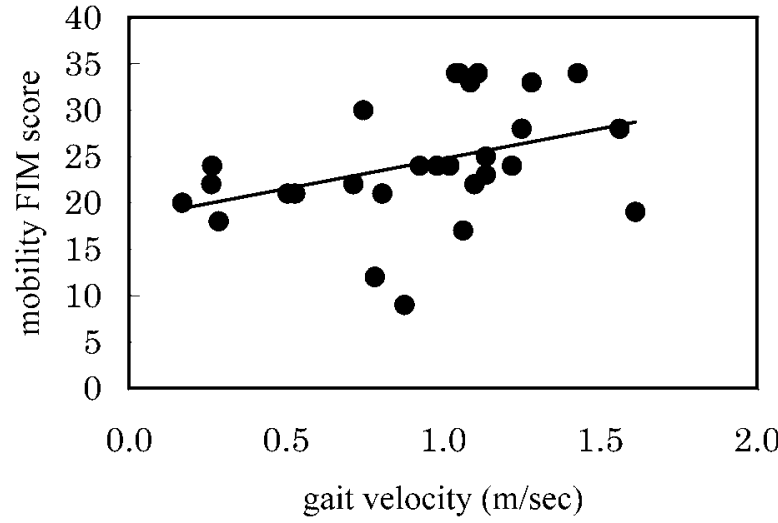

Fig. 1. Correlation between the mobility FIM score and gait velocity.

assess body composition and functional performance with a group of subacute male stroke survivors, and to evaluate their relationship. Our results are consistent with prior literature regarding the importance of such variables as age, gender, severity of illness, and baseline level of function.

In this study, the FIM subscores failed to correlate with BMI but correlated significantly with the BRS and LBM. Only the mobility FIM subscore correlated significantly with gait velocity. However, low total FIM scores were also obtained for subjects who achieved high levels of gait velocity. This may reflect not only the broadness of the function evaluated by FIM, but also the lack of sensitivity of this scale when small differences exist ${ }^{10)}$. This finding corroborates the need to narrowly define measurement constructs of hemiparetic stroke patients.

Gait velocity is reduced with hemiparesis. In our study, there were subjects whose mean gait velocity was $0.93 \pm 0.38 \mathrm{~m} / \mathrm{sec}$ ranging from 0.17 to $1.61 \mathrm{~m} /$ sec. The gait velocity of these subacute patients revealed important deficits in performance. However, even in the subacute phases, the variability in the gait velocity can be large, as demonstrated this study (Fig. 1).

One study indicated that the gait velocity of a group of 44 subjects $(50.5 \pm 19.0$ days post stroke) was $0.30 \mathrm{~m} / \mathrm{sec}^{12)}$. Richards et al. ${ }^{15)}$ reported that the walking velocity in different studies of stroke survivors ranged from 0.25 to $0.63 \mathrm{~m} / \mathrm{sec}$. In another study, a comparison of the gait velocity of acute stroke patients $(n=42)$ with gender- and agematched controls $(n=42)$ suggested that the group mean for the stroke patients of $0.45 \mathrm{~m} / \mathrm{sec}$ was significantly lower than the group mean of $1.15 \mathrm{~m} /$ $\mathrm{sec}$ for control subjects $(p<0.0001)^{13)}$. Independent gait velocity has been defined as a gait velocity of 0.80 to $1.50 \mathrm{~m} / \mathrm{sec}^{11,14)}$. A gait velocity of $1.2 \mathrm{~m} /$ sec has been reported to be required to cross a commercial street according to the traffic signal timing ${ }^{11)}$.

Bioelectric impedance analysis can be used to assess body composition and may be useful for nutritional assessment of patients who have undergone hemiplegia. Malnutrition and weight loss are common after stroke. Therefore the great variability in weight change was unexpected. Substantial loss of BF may increase the risk of fracture due to decreased energy absorption in accidental falls ${ }^{18)}$. A potential confounding factor is interference of measurements from edema. A change in hydration may theoretically interfere with the measurement of LBM, because LBM contains fluid as well as skeletal muscle ${ }^{17}$ ). Therefore, we may have underestimated the actual loss of LBM after stroke.

The result obtained in this study concerning the mean $\% \mathrm{BF}$ was lower than those of previous studies $\left(31.4 \pm 9.8 \%{ }^{16)}, 29.9 \pm 9.8 \%{ }^{17)}\right)$. The differences between those results and our result might be explained in part by the selection of patients and by the study design. Our results indicate that arm body water is significantly lower in the affected limb than in the unaffected side in subacute hemiparetic stroke patients. This may account for why the degree of paralysis in the upper limbs of our subjects due to physical inactivity is lower in the affected arm than in the unaffected arm. However, it is necessary to walk with both the affected leg and the unaffected leg. Our results support those of Iversen et al. ${ }^{19)}$, who reported that dual-energy Xray absorptiometry showed an approximate $10 \%$ and 3\% lower lean content of the arms and legs, respectively, in a small sample of stroke patients $(n=15)$.

In stroke patients, a previous study found that loss of BMC on the affected side after stroke was marked and was associated with a significant difference in body composition according to side, with decreased LBM and increased BF in the affected side as opposed to the unaffected side. Concomitantly, no correlation was seen between loss of BMC or change in body composition and the degree of paresis ${ }^{19)}$. Further studies are needed to 
determine the magnitude of hemiatrophy of the limbs in hemiparetic patients. The main limitation of our study was the lack of a study group large enough to perform statistical analysis of the data based on the phase of stroke or the level of functional motor control.

\section{REFERENCES}

1) Maulden SA, Gassaway J, Horn SD, et al.: Timing of initiation of rehabilitation after stroke. Arch Phys Med Rehabil, 2005, 86 (Suppl 2): S34-40.

2) Kelly JO, Kilbreath SL, Davis GM, et al.: Cardiorespiratory fitness and walking ability in subacute stroke patients. Arch Phys Med Rehabil, 2003, 84: 1780-1785.

3) Potempa K, Lopez M, Braun LT, et al.: Physiological outcomes of aerobic exercise training in hemiparetic stroke patients. Stroke, 1995, 26: 101-105.

4) Monga TN, Deforge DA, Williams J, et al.: Cardiovascular responses to acute exercise in patients with cerebrovascular accidents. Arch Phys Med Rehabil, 1988, 69: 937-940.

5) Eng JJ, Chu KS, Dawson AS, et al.: Functional walk tests in individuals with stroke: relation to perceived exertion and myocardial exertion. Stroke, 2002, 33: 756-761.

6) Brunnstrom S: Motor testing procedures in hemiplegia: based on sequential recovery stages. Phys Ther, 1966, 46: 357-375.

7) Sakai T, Tanaka K, Holland GJ: Functional and locomotive characteristics of stroke survivors in Japanese community-based rehabilitation. Am J Phys Med Rehabil, 2002, 81: 675-683.

8) Casanova Roman M, Rodriguez Ruiz I, Rico de Cos S: Body composition analysis using bioelectrical and anthropometric parameters. An Pediatr (Barc), 2004, 61: $23-31$.

9) Cunha IT, Lim PA, Henson H, et al.: Performancebased gait tests for acute stroke patients. Am J Phys Med Rehabil, 2002, 81: 848-856.

10) Bunch WH, Dvonch VM: The "value" of functional independence measure scores. Am J Phys Med Rehabil, 1994, 73: 40-43.

11) Perry J, Garrett M, Gronley JK, et al.: Classification of walking handicap in the stroke population. Stroke, 1995, 26: 982-989.

12) Richards CL, Malouin F, Dean C: Gait in stroke: assessment and rehabilitation. Clin Geriatr Med, 1999, 15: 833-855.

13) Goldie PA, Matyas TA, Evans OM: Deficit and change in gait velocity during rehabilitation after stroke. Arch Phys Med Rehabil, 1996, 77: 1074-1082.

14) Friedman PJ: Gait recovery after hemiplegic stroke. Int Disabil Stud, 1990, 12: 119-122.

15) Richards CL, Malouin F, Wood-Dauphinee S, et al.: Task-specific physical therapy for optimization of gait recovery in acute stroke patients. Arch Phys Med Rehabil, 1993, 74: 612-620.

16) Ryan AS, Dobrovolny CL, Smith GV, et al.: Hemiparetic muscle atrophy and increased intramuscular fat in stroke patients. Arch Phys Med Rehabil, 2002, 83: 1703-1707.

17) Ramnemark A, Nyberg L, Lorentzon R, et al.: Hemiosteoporosis after severe stroke, independent of changes in body composition and weight. Stroke, 1999, 30: 755-760.

18) Slemenda CW: Body composition and skeletal density: mechanical loading or something more? J Clin Endocrinol Metab, 1995, 80: 1761-1763.

19) Iversen $E$, Hassager $C$, Christiansen $C$ : The effect of hemiplegia on bone mass and soft tissue body composition. Acta Neurol Scand, 1989, 79: 155-159. 